\title{
THE PHYSICS OF THE INTERSTELLAR MATTER
}

\author{
G. B. FIELD \\ Center for Astrophysics, Harvard College Observatory and \\ Smithsonian Astrophysical Observatory, Cambridge, Mass., U.S.A.
}

\section{Introduction}

Some of the most striking recent discoveries about interstellar matter involve molecules. It has been known for a long time that there are atoms and ions in space mainly hydrogen and helium, of course - but also heavier elements like sodium and calcium. In addition, there are solid particles of dust, about $10^{-5} \mathrm{~cm}$ across, which must be composed of heavier elements, as hydrogen and helium cannot condense under interstellar conditions.

In 1972, the Orbiting Astronomical Observatory-3, which employs an $80-\mathrm{cm}$ telescope at wavelengths between $1000 \AA$ and $3000 \AA$, was launched in the United States and put into operation. In the ensuing year, it has demonstrated that much of the interstellar medium is composed of hydrogen molecules. This result, based upon the observation of Lyman-band absorption in the spectrum of early-type stars, had been anticipated by a rocket observation of $\mathrm{H}_{2}$ by Carruthers in 1970 .

The same OAO-3 instrument observed resonance lines of many cosmically abundant elements, and found that these elements often appear to be less abundant in interstellar space than in the solar system, relative to hydrogen. As young stars born recently from the interstellar medium do not show this effect, the heavy elements must in fact be present in some other form. Here I will argue that the heavy elements are largely locked up in the form of the dust and, further, that dust is critical for the formation of the molecules in interstellar space.

It is appropriate that the Orbiting Astronomical Observatory-3 which made these discoveries has been named in honor of Copernicus, the Polish astronomer we honor here on the 500th anniversary of his birth. Just as his discoveries were revolutionary for the understanding of the solar system, those made using the Observatory named in his honor have been revolutionary for the understanding of the Galaxy.

\section{Interstellar Dust}

The chemical composition of interstellar dust has been hotly debated for many years. A resolution of this question was not possible as long as astronomers were limited by the information available in the visible region, because there the extinction by dust exhibits only a slow variation with wavelength. This can be matched with several different materials which might be expected to be present, such as ice, silicates, graphite, metallic iron, etc. Recent exploration of the infrared and ultraviolet bands has provided critical new information, however. 


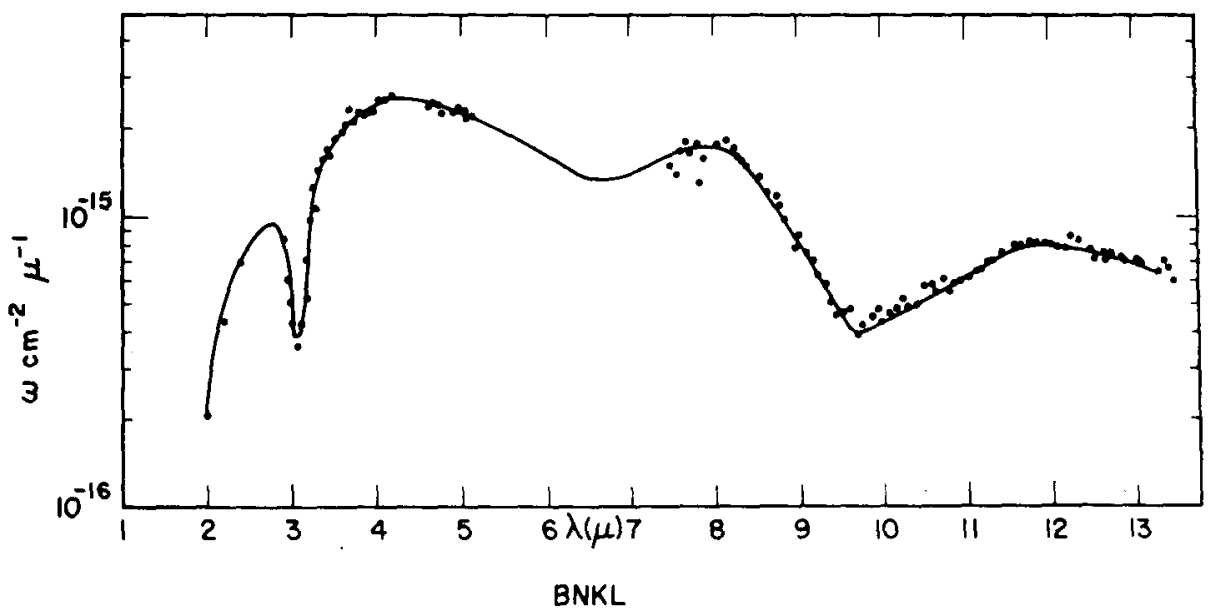

Fig. 1. Spectrum of the Becklin-Neugebauer point infrared source in Orion, due to Gillett et al. (1972). Note the two absorption bands, at $3.1 \mu$ and $9.7 \mu$, characteristic of water ice and of silicates.

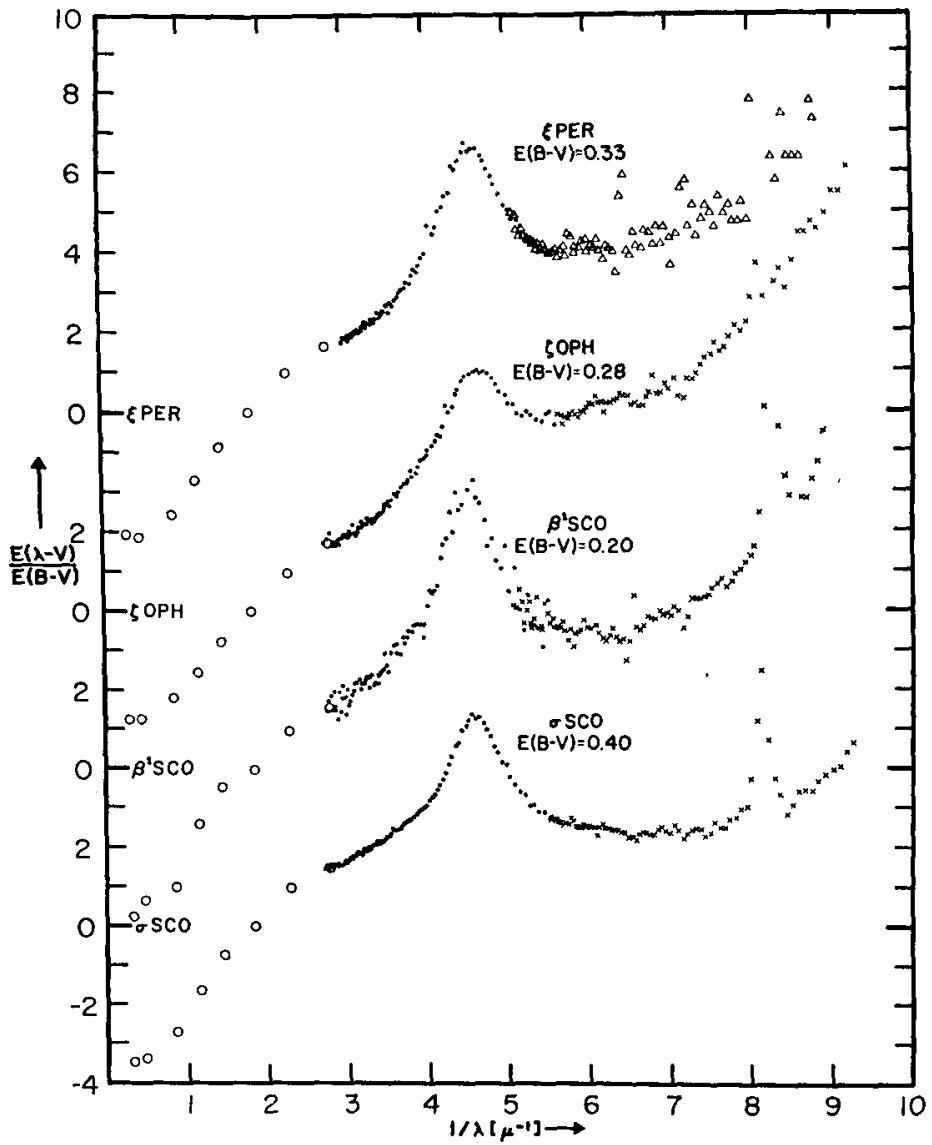

Fig. 2. Interstellar extinction curves for four stars, with normalized absorption increasing upwards (Bless and Savage, 1972). The absorption feature at $2200 \AA\left(\lambda^{-1}=4.6 \mu^{-1}\right)$ has been identified as graphite. 
Figure 1 shows the infrared spectrum of the infrared point source in Orion. Two absorption features are seen: that at 3.1- $\mu$ wavelength is characteristic of water ice, while that at 9.7- $\mu$ wavelength is characteristic of silicates such as $\mathrm{MgSiO}_{3}$ or $\mathrm{Mg}_{2} \mathrm{SiO}_{4}$. Figure 2 shows the interstellar extinction curve in the ultraviolet for several early-type stars. One notes an absorption feature at $2200 \AA$, which is seen in each of the about 200 stars observed. This feature is characteristic of small particles of graphite. Recently, a feature of silicon carbide has been observed at $11.5 \mu$. Thus, there is direct evidence for particles containing ice, silicates, graphite and silicon carbide in interstellar space.

How do such particles originate? Attempts to explain their origin in interstellar space have failed; the time scales for atoms to collide and stick are too large at the very low gas densities involved, $10-100 \mathrm{~cm}^{-3}$. It was suggested, rather, that dust grains condense in the relatively high-density conditions prevailing in the outer envelopes of stars, provided such envelopes are cool enough. The condensation process has been studied theoretically, with the results in Table I. One sees that in an oxygen-rich atmosphere the most abundant elements (C, N, O, Mg, Si, and $\mathrm{Fe}$ ) condense as methane, water, ammonia, magnesium silicates, and metallic iron, while in a carbon-rich atmosphere, one expects graphite, silicon carbide, and iron carbide. Evidence that condensation actually does occur in some stars is provided by infrared spectra in which the $9.7-\mu$ feature appears in emission, the interpretation being that the circumstellar dust is heated by the star and that it emits in the 9.7- $\mu$ band, where its absorptivity is high. For this to work, the dust must be near the star.

Independent confirmation of circumstellar dust has been obtained through polarization studies. While most stars show a fixed polarization due to aligned dust particles in interstellar space, many late-type stars also show variable polarization which can be explained only if the responsible dust is very close to the star. Furthermore,

TABLE I

Condensation temperatures ${ }^{a}$

\begin{tabular}{|c|c|c|c|}
\hline Stage & $\begin{array}{l}\text { Temperature } \\
\text { (K) }\end{array}$ & Condensates & $\begin{array}{l}\text { Elements } \\
\text { removed }\end{array}$ \\
\hline \multirow[t]{3}{*}{1} & $1400-1600$ & $\mathrm{CaTiO}_{3}$ & $\mathrm{Ti}$ \\
\hline & & $\begin{array}{llll}\mathrm{Mg}_{2} \mathrm{Al}_{2} \mathrm{O}_{4} & \mathrm{Al}_{2} \mathrm{SiO}_{3} & \mathrm{CaAl}_{2} \mathrm{Si}_{2} \mathrm{O}_{8}\end{array}$ & Al \\
\hline & & $\begin{array}{llll}\mathrm{CaMgSi}_{2} \mathrm{O}_{6} & \mathrm{Ca}_{2} \mathrm{SiO}_{4} & \mathrm{CaSiO}_{3}\end{array}$ & $\mathrm{Ca}$ \\
\hline 2 & $1220-1320$ & $\begin{array}{lll}\mathrm{MgSiO}_{3} & \mathrm{Mg}_{2} \mathrm{SiO}_{4} & \mathrm{BeAl}_{2} \mathrm{O}_{4}\end{array}$ & $\mathrm{Si}, \mathrm{Mg}, \mathrm{Be}$ \\
\hline 3 & 1280 & Metallic $\mathrm{Fe} \mathrm{Ni}$ & $\mathrm{Fe}, \mathrm{Ni}$ \\
\hline 4 & 1210 & $\mathrm{MnSiO}_{3}$ & Mn \\
\hline 5 & $970-1070$ & Alkali Silicates & $\mathrm{Na}, \mathrm{K}, \mathrm{Rb}$ \\
\hline 6 & $600-700$ & $\mathrm{FeS} \mathrm{NaBO}_{2}$ & $\mathrm{~S}, \mathrm{~B}$ \\
\hline 7 & 180 & $\mathrm{H}_{2} \mathrm{O}$ & $\mathrm{O}$ \\
\hline 8 & 120 & $\mathrm{NH}_{3} . \mathrm{H}_{2} \mathrm{O}$ & $\mathbf{N}$ \\
\hline 9 & 75 & $\mathrm{CH}_{4} . \mathrm{XH}_{2} \mathrm{O}$ & $\mathrm{C}$ \\
\hline 10 & 25 & Ar(solid) & Ar \\
\hline
\end{tabular}

a See, e.g., Lewis (1972). 
many late-type stars are known from spectroscopic studies to be losing gaseous materìal at substantial rates. Hence, one concludes that some late-type stars are losing material and that much of the associated heavy elements has condensed to form dust. Quantitatively, it appears that the rate at which dust is being ejected into space by this mechanism is adequate to account for the amount of interstellar dust which is observed.

\section{Depletion of Gas-Phase Elements}

If it is really true that heavy elements which are injected into space by stars have condensed partly into dust grains, one would expect this effect to show up when atoms and ions are sought spectroscopically in the gas phase. The Copernicus satellite telescope observed resonance lines of interstellar $\mathrm{H}, \mathrm{B}, \mathrm{C}, \mathrm{N}, \mathrm{O}, \mathrm{Mg}, \mathrm{Si}, \mathrm{P}, \mathrm{S}, \mathrm{Cl}, \mathrm{Ar}$, $\mathrm{Mn}$, and $\mathrm{Fe}$, while previous ground-based measurements gave data on $\mathrm{Li}, \mathrm{Be}, \mathrm{Na}, \mathrm{Al}$, $\mathrm{K}, \mathrm{Ca}$, and $\mathrm{Ti}$. In the direction of the $09.5 \mathrm{star} \zeta \mathrm{Oph}$, there is a wealth of information, including a measurement of the ratio of atomic calcium to ionized calcium in the cloud between us and $\zeta \mathrm{Oph}$. This ratio permits one to estimate the electron density in the cloud and, therefore, to calculate the abundances of certain other ions which are not directly observed.

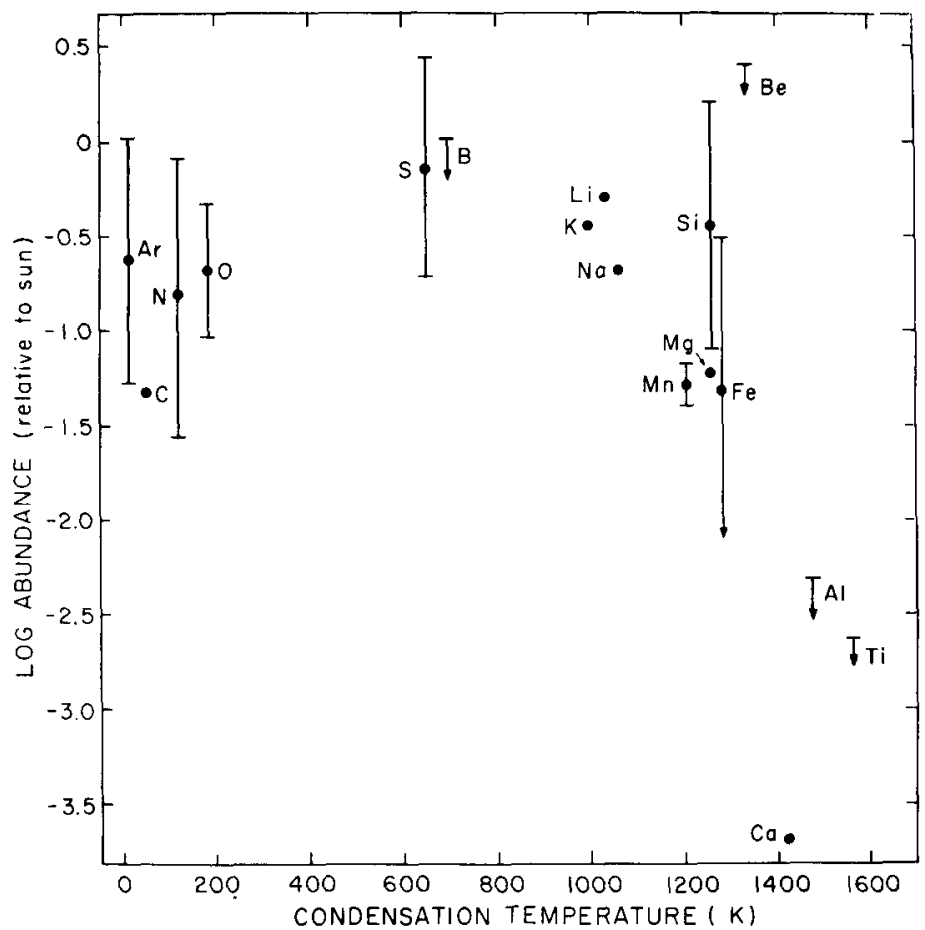

Fig. 3. Abundances toward $\zeta$ Oph on a logarithmic scale, relative to solar-system values (Field, 1974). The horizontal axis is condensation temperature, defined as that temperature at which the element condenses into solid particles, according to the sequence of Table I. Note the tendency for the most condensible elements to be most depleted, suggesting that they are condensed into dust grains. 
The results, expressed as column densities of various gas-phase elements relative to those expected if the abundances are normal, are shown in Figure 3. It is instructive to plot the observed abundances against the theoretical condensation temperatures given in Table I. The data indicate that those elements which should condense at the highest temperatures (notably $\mathrm{Al}, \mathrm{Ca}$, and $\mathrm{Ti}$ ) are most underabundant. There is a general tendency for the underabundance to correlate with condensation temperature, suggesting that the heavy elements have actually condensed into dust grains, this process being most effective for the highest temperature condensates. This would be expected if, for example, the condensation process occurs while gas is flowing away from a star, for high-temperature materials would condense near the star, where the gas density is high. Low-temperature materials would condense only far away from the star, where the gas density is so low that the process would not be completed.

From Figure 3 one sees that the abundant elements $\mathrm{C}, \mathrm{N}, \mathrm{O}, \mathrm{Mg}, \mathrm{Si}$, and Fe are all depleted in the gas phase by substantial factors. One may wonder whether these elements are really locked up in dust or, rather, are present in some other form, such as gas molecules. One finds spectroscopically that while gas molecules are indeed present in the $\zeta$ Oph cloud, they have much too low an abundance to account for the observed depletion of atoms and ions. We conclude that these elements are in the dust.

One is, therefore, led to consider models of grains which are composed of these elements, in those forms predicted by the condensation theory, notably graphite, methane, ammonia, water, magnesium silicates, silicon, carbide, and metallic iron. If one confines attention to spherical grains (perhaps an unreasonable assumption), one finds that one can just explain the observed visual extinction if all the missing abundant elements are in the form of these materials. Even more significant, quantitative agreement is obtained with the observed silicate absorption in the Orion infrared source (Figure 1) and with the observed graphite absorption in $\zeta$ Oph (Figure 2).

Difficulty is encountered, however, with water ice. If all the missing oxygen which is not chemically combined in silicates is assumed to be in water, the predicted 3.1- $\mu$ band is about ten times stronger than the observed one (Figure 1). This problem is discussed further below.

\section{Interaction of Gas and Dust}

Figure 3 reveals an apparent anomaly. Depletion factors decrease steadily with decreasing condensation temperature down to $650 \mathrm{~K}$, where $S$ is depleted by a factor less than 5. Yet the very low-temperature condensates $\mathrm{C}, \mathrm{N}, \mathrm{O}$, and Ar are depleted by even greater factors. This suggests that a different process may be involved for the low-temperature condensates; we tentatively identify this to be the slow accumulation of ice mantles on the dust grains after they have become part of the interstellar cloud.

Does this actually occur?

To answer this question requires an understanding of the surface physics of graphite, 
iron, and silicate dust grains, which is not available at present. One can make some general points, however.

In a typical interstellar cloud, a hydrogen atom collides with a dust grain about once every 10 million years. Very likely it will stick there, as it is bound by van der Waals forces. Because hydrogen is the dominant constituent of interstellar gas, a layer of $\mathrm{H}$ atoms builds up on the grain. Hence the $\mathrm{H}$ atoms on the grain are closely surrounded by other $\mathrm{H}$ atoms, and there is a fair probability that they will react to form $\mathrm{H}_{2}$ via

$$
\mathrm{H}+\mathrm{H} \rightarrow \mathrm{H}_{2}+4.5 \mathrm{eV} .
$$

The $4.5-\mathrm{eV}$ energy released goes either into heating the glain or into kinetic energy of the molecule. As it is much greater than the binding energy to the grain, the newly-formed $\mathrm{H}_{2}$ probably is ejected at once. We discuss the fate of the resulting $\mathrm{H}_{2}$ molecules further below.

Other atoms, particularly the abundant ones $\mathrm{C}, \mathrm{N}$, and $\mathrm{O}$, also collide with dust grains about every 10 million years, and it is believed that they, too, will stick. But these atoms are surrounded not by others of the same type, but by the $\mathrm{H}$ atoms in the layer described above. Hence, they are likely to react with $\mathrm{H}$ to form the radicals $\mathrm{CH}, \mathrm{OH}$, and $\mathrm{NH}$.

At this point, it is uncertain what happens. On the one hand, the radicals may be ejected at once, like $\mathrm{H}_{2}$, and, in fact, gas-phase $\mathrm{CH}$ and $\mathrm{OH}$ have been observed. On the other hand, their much greater mass may reduce the ejection probability, so that they stay on the grain. In the latter event, they will react further, ultimately forming the saturated molecules $\mathrm{CH}_{4}, \mathrm{NH}_{3}$, and $\mathrm{H}_{2} \mathrm{O}$. The latter molecules, accumulated on the grain, would constitute the ice mantle I spoke of earlier and would explain the observed depletion of $\mathrm{C}, \mathrm{N}$, and $\mathrm{O}$.

Does mantle formation actually occur in interstellar space?

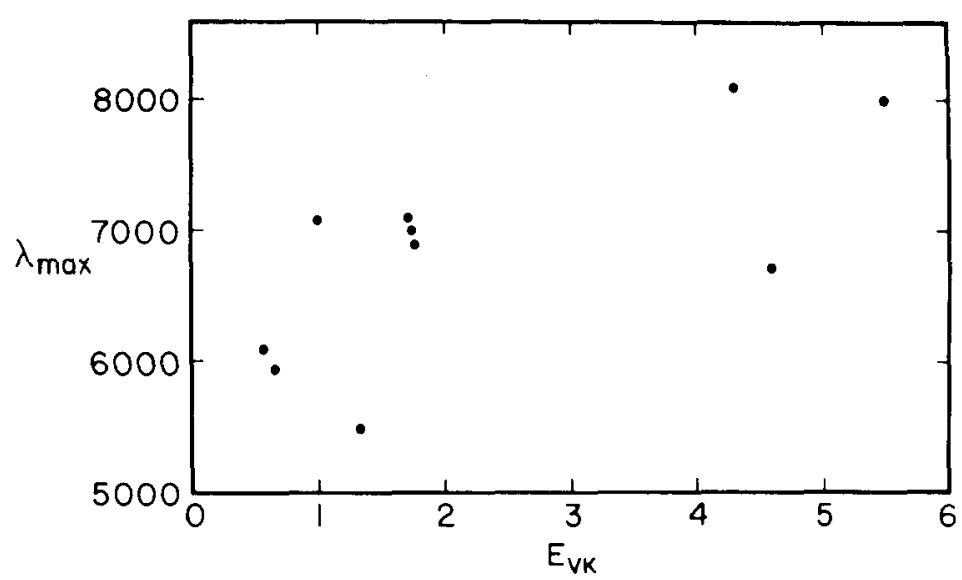

Fig. 4. The wavelength of maximum polarization, plotted against the extinction $A_{V}-A_{K} \simeq A_{V}$, for various stars shining through the dark cloud near $Q$ Ophiuchi (Carrasco et al., 1973). 
The evidence on this point is often ambiguous, but recently, observations of the well-known dark cloud near $\varrho$ Oph indicate strongly that the dust in this cloud is accreting mantles. The density in the cloud varies from about $10 \mathrm{~cm}^{-3} 6 \mathrm{pc}$ from the center to about $1000 \mathrm{~cm}^{-3} 0.4 \mathrm{pc}$ from the center. The extinction of stars seen through the cloud also increases inward. The light from stars shining through the cloud is polarized and the degree of polarization varies with wavelength in the usual way, reaching a maximum at $\lambda_{\max }$ located in the observable range. Normally $\lambda_{\max }$ is about $5400 \AA$, but in this cloud, it rises from about that value in the outer parts of the cloud to about $8000 \AA$ in the inner part of the cloud (Figure 4). Theory indicates that $\lambda_{\max }$ should be roughly proportional to particle size, so this indicates that the size has increased $60 \%$ in going from the outer to the inner part of the cloud. This can be explained if substantial amounts of $\mathrm{C}, \mathrm{N}$, and $\mathrm{O}$ have accreted on to the dust grains in the cloud. The rate at which this happens is proportional to density, so it is natural that the dense inner parts of the clouds, where $A_{V}$ is larger, should show the larger effect. I conclude that accretion of mantles occurs in at least some clouds.

However, one is left with the problem of the weak 3.1- $\mu$ band of $\mathrm{H}_{2} \mathrm{O}$ ice. One may speculate here that absorption of stellar ultraviolet light may lead to further chemical changes in the mantle. Each absorption dissociates a molecule, momentarily forming a radical which reacts rapidly with neighboring molecules. Such processes have been observed in the laboratory to yield complex molecules at a rate which would give a time scale of a few million years if translated to interstellar conditions. Thus, most of the oxygen may be combined into complex organic molecules and the fraction of oxygen combined in water may be small, as observed.

We referred above to $\mathrm{H}_{2}$ molecules forming on grains and being ejected into the interstellar medium. This process is very important in the Galaxy, for it is the only known way to efficiently catalyze the formation of $\mathrm{H}_{2}$ molecules from $\mathrm{H}$ atoms in space. The reason for this is that if two $\mathrm{H}$ atoms approach each other in the gas phase, there is no third body to take up the energy of formation, so that the atoms may form a bound system. Consequently, the only way that a molecule can form is by the emission of radiation, which for a homonuclear molecule like $\mathrm{H}_{2}$ requires quadrupole emission. This is so weak that $\mathbf{H}_{2}$ molecules cannot form in appreciable amounts in this way. Thus, the mere fact that $\mathbf{H}_{2}$ is observed in interstellar space indicates that the $\mathrm{H}_{2}$ formation is being catalyzed by dust grains.

Of course, $\mathrm{H}_{2}$ can also be destroyed in interstellar space. The most important process is

$$
\begin{aligned}
& \mathrm{H}_{2}\left(X^{\prime} \Sigma_{g}^{+}\right)+h v \rightarrow \mathrm{H}_{2}\left(\mathrm{~B}^{\prime} \Sigma_{u}^{+}\right) \\
& \rightarrow \mathrm{H}_{2}^{*}\left(X^{\prime} \Sigma_{g}^{+}\right) \rightarrow \mathrm{H}+\mathrm{H},
\end{aligned}
$$

where $\mathrm{H}_{2}^{*}$ indicates vibrational continuum and where $h v$ is a photon of the Lyman band $(\lambda<1108 \AA)$. In low-density regions, this occurs every 300 years, a much smaller time scale than the millions of years it takes to form $\mathbf{H}_{2}$. Hence, in unshielded space, one expects that $\mathrm{H}_{2} / \mathrm{H} \ll 1$. 
However, in a denser cloud, the newly formed $\mathrm{H}_{2}$ molecules on the outside of the cloud absorb the Lyman-band photons before they reach the interior and the molecules inside are thereby shielded. The theory of this effect yields the results in Figure 5, where I have also plotted some of the observational data from Copernicus. There is a qualitative agreement, particularly if one includes slightly lower cloud masses. The theory predicts that in unreddened stars, presumably seen through the intercloud medium, the fractional $\mathrm{H}_{2}$ abundance should be $4 \times 10^{-8}$. The observations give upper limits of about this value. Therefore, the observations agree quite well with the theory that $\mathrm{H}_{2}$ is catalyzed by grains and destroyed by ultraviolet light.

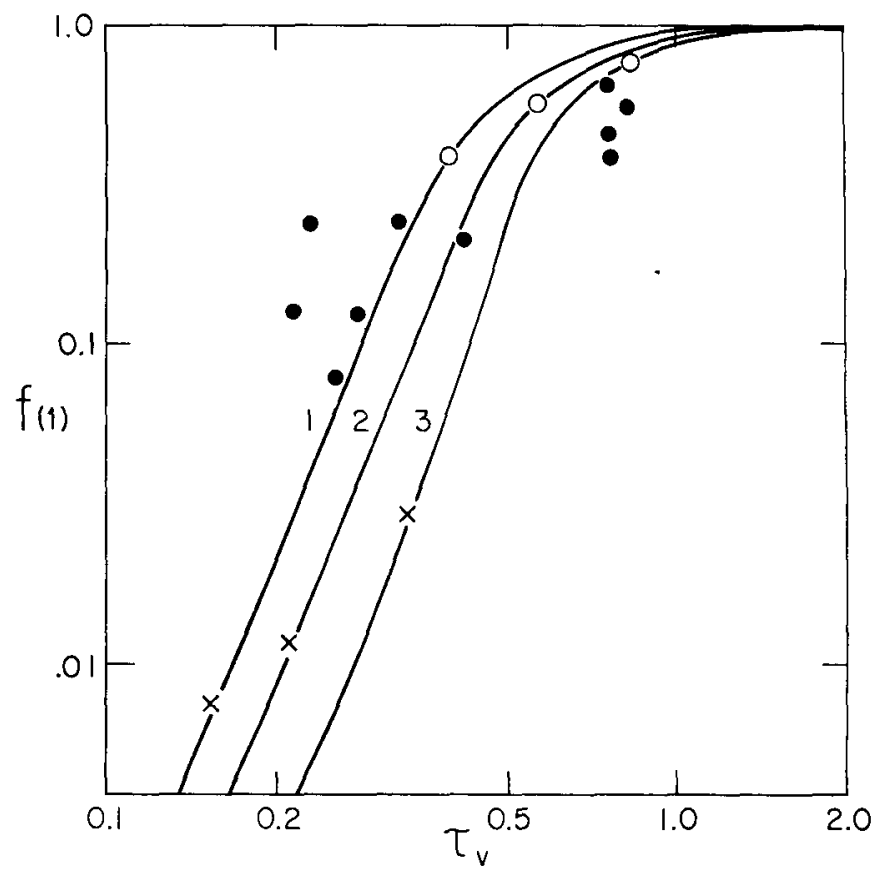

Fig. 5. Fractional abundance of $\mathrm{H}_{2}$ in interstellar clouds, plotted against optical depth to the cloud center in the visible, $\tau_{V}$, calculated by Hollenbach et al. (1971). One can show that on the average $\tau_{V}=\frac{3}{4} A_{V}$, where $A_{V}$ is the extinction through the cloud at a random point. Since $A_{V}=3 E_{B-V}$, $\tau_{V}=2.25 E_{B_{-} V}$ and the observations may be plotted as shown by the filled circles. The observations are from Copernicus (Spitzer et al., 1973).

This has immediate consequences for the interstellar medium. It has been estimated that up to $40 \%$ of all interstellar gas may be in clouds which are thick enough that they are mostly $\mathrm{H}_{2}$. Hence, previous estimates of the mean density of the interstellar medium may be increased as much as $75 \%$.

To summarize this section, I have described how molecules can form upon grains and then either enter the gas phase or accumulate as part of a mantle. That the first process occurs is demonstrated by the discovery of large amounts of $\mathbf{H}_{2}$ by 
Copernicus. That the second process occurs is indicated by the depletion of $\mathrm{C}, \mathrm{N}$, and $\mathrm{O}$ found by Copernicus and by the systematic variations with radius in the dark cloud near $\varrho$ Oph.

\section{Molecules}

We have seen that $\mathrm{H}_{2}$ is catalyzed by dust grains. But radio astronomers have observed the spectral lines of a large variety of molecules (Table II), ranging from diatomics like $\mathrm{OH}$, to seven-atom molecules like acetaldehyde $\left(\mathrm{CH}_{3} \mathrm{CHO}\right)$. Several diatomics were discovered optically many years ago by their absorption lines in stars, but all the complex molecules have been discovered quite recently by radio astronomers.

TABLE II

Molecules observed in the interstellar medium 1973

\begin{tabular}{|c|c|}
\hline $\mathrm{H}_{2}$ & Molecular hydrogen \\
\hline $\mathrm{CH}^{+}$ & Methylidyne (ion) \\
\hline $\mathrm{CH}$ & Methylidyne \\
\hline $\mathrm{CN}$ & Cyanogen radical \\
\hline $\mathrm{OH}$ & Hydroxyl radical \\
\hline $\mathrm{NH}_{3}$ & Ammonia \\
\hline $\mathrm{H}_{2} \mathrm{O}$ & Water \\
\hline $\mathrm{H}_{2} \mathrm{CO}$ & Formaldehyde \\
\hline $\mathrm{CO}$ & Carbon monoxide \\
\hline $\mathrm{HCN}$ & Hydrogen cyanide \\
\hline $\mathrm{HCO}^{+}(?)$ & Formyl radical (ion) \\
\hline $\mathrm{HC}_{3} \mathrm{~N}$ & Cyanoacetylene \\
\hline $\mathrm{CH}_{3} \mathrm{OH}$ & Methyl alcohol \\
\hline $\mathrm{HCOOH}$ & Formic acid \\
\hline CS & Carbon monosulfide \\
\hline $\mathrm{NH}_{2} \mathrm{CHO}$ & Formamide \\
\hline OCS & Carbonyl sulfide \\
\hline $\mathrm{SiO}$ & Silicon monoxide \\
\hline $\mathrm{CH}_{3} \mathrm{CN}$ & Methyl cyanide \\
\hline HNCO & Isocyanic acid \\
\hline HNC(?) & Hydrogen isocyanide \\
\hline $\mathrm{CH}_{3} \mathrm{CCH}$ & Methyl acetylene \\
\hline $\mathrm{CH}_{3} \mathrm{CHO}$ & Acetaldehyde \\
\hline $\mathrm{H}_{2} \mathrm{CS}$ & Thioformaldehyde \\
\hline $\mathrm{H}_{2} \mathrm{~S}$ & Hydrogen sulfide \\
\hline $\mathrm{CH}_{2} \mathrm{NH}$ & Methanimine \\
\hline
\end{tabular}

The fact that some of the complex interstellar molecules resemble fragments of organic molecules which are important in biological processes - such as amino acids - has led to speculation that they are produced by living organisms beyond the Earth. As a Copernican, I am open to the idea that the Earth is not the unique locus of life in the universe. But there is no need to postulate life in space to understand the molecules observed.

Dust grains can also catalyze other molecules - at least simple ones like $\mathrm{OH}$. 
However, it is not clear that they can also catalyze more complex ones. Hence there is interest in a recent theory which demonstrates that it is possible to build up more complex molecules by gas-phase reactions. The key to this theory is the ionization of $\mathrm{H}_{2}$ molecules by cosmic rays, which are believed to penetrate all space in the Galaxy. The important ionization reaction is

$$
\mathbf{H}_{2}+\mathbf{C R} \rightarrow \mathbf{H}_{2}^{+}+e^{-}+\mathbf{C R} .
$$

This process occurs about once every $3 \times 10^{9}$ yr to each $\mathrm{H}_{2}$ molecule. Neither the $\mathrm{H}_{2}^{+}$nor $e^{-}$density ever becomes large, so recombination is very slow. Instead, the $\mathrm{H}_{2}^{+}$reacts rapidly with $\mathrm{H}_{2}$ :

$$
\mathrm{H}_{2}^{+}+\mathrm{H}_{2} \rightarrow \mathrm{H}_{3}^{+}+\mathrm{H} \text {. }
$$

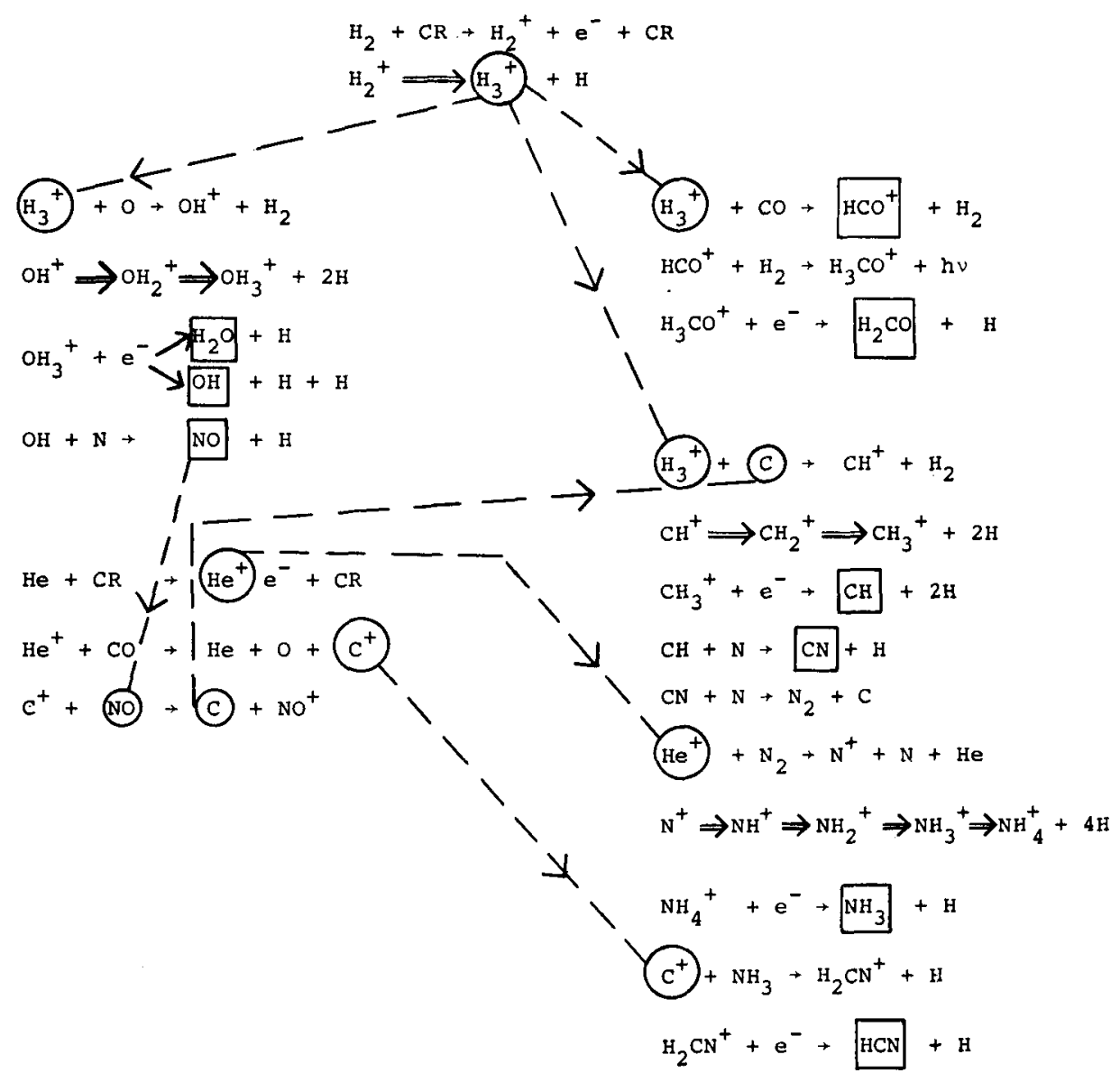

Fig. 6. Ion-molecule reactions in dark clouds (Herbst and Klemperer, 1973). The reactions are initiated by ionization of $\mathrm{H}_{2}$ (top) and $\mathrm{He}$ (bottom left) and result in the species denoted by a box. The ingredients are $\mathrm{H}_{2}, \mathrm{He}, \mathrm{CO}, \mathrm{N}, \mathrm{O}$, and $100-\mathrm{MeV}$ cosmic rays (CR). The symbol $\Rightarrow$ refers to reaction with $\mathrm{H}_{2}$, e.g., $\mathrm{A}^{+}+\mathrm{H}_{2} \rightarrow \mathrm{AH}^{+}+\mathrm{H}$. The species produced include $\mathrm{CH}, \mathrm{OH}, \mathrm{CN}, \mathrm{NO}, \mathrm{H}_{2} \mathrm{O}$, $\mathrm{NH}_{3}, \mathrm{HCO}^{+}, \mathrm{H}_{2} \mathrm{CO}$, and $\mathrm{HCN}$, of which all but $\mathrm{HCO}^{+}$and $\mathrm{NO}$ have definitely been identified in interstellar space. $\left(\mathrm{HCO}^{+}\right.$has been tentatively identified.) 
TABLE III

Comparison of observed abundances with a theory of molecule formation ${ }^{\mathrm{a}}$

\begin{tabular}{lcrr}
\hline \multicolumn{4}{l}{$\log N_{\text {obs }}-\log N_{\text {th o or }}$} \\
\hline Molecule & Dark clouds & Orion A & Sgr B2 \\
\hline $\mathrm{OH}$ & +1.1 & -0.1 & +0.4 \\
$\mathrm{CN}$ & - & 0.0 & - \\
$\mathrm{NO}$ & $<+0.5$ & $<+1.2$ & $<+0.5$ \\
$\mathrm{NH}_{3}$ & - & - & - \\
$\mathrm{HCO}^{+}$ & - & -0.3 & - \\
$\mathrm{H}_{2} \mathrm{CO}$ & -0.5 & +0.5 & -0.4 \\
$\mathrm{HCN}$ & - & +0.5 & - \\
\hline
\end{tabular}

a Based on Herbst and Klemperer (1973).

b $10 \times$ local cosmic-ray flux.

Now $\mathrm{H}_{3}^{+}$reacts rapidly with $\mathrm{O}$ to form $\mathrm{OH}^{+}$, and a further chain of reactions with $\mathrm{H}_{2}$ makes $\mathrm{H}_{2} \mathrm{O}$ and $\mathrm{OH}$. None of these reactions requires emission of radiation. Furthermore, because the ions involved exert a strong attractive force, there is no activation energy. Hence, these 'ion-molecule' reactions are much more rapid than reactions between neutrals.

It has been found observationally that in dark clouds, where hydrogen is mostly $\mathrm{H}_{2}$, much of the carbon is in the form of $\mathrm{CO}$. Hence the theory has been applied to dark clouds, where the principal ingredients available are $\mathrm{H}_{2}, \mathrm{He}, \mathrm{CO}, \mathrm{N}$, and $\mathrm{O}$. Figure 6 summarizes the key reactions which occur. The products include $\mathrm{CH}, \mathrm{OH}$, $\mathrm{CN}, \mathrm{NO}, \mathrm{H}_{2} \mathrm{O}, \mathrm{NH}_{3}, \mathrm{HCO}^{+}, \mathrm{H}_{2} \mathrm{CO}$, and $\mathrm{HCN}$. Seven of these molecules, and perhaps one more $\left(\mathrm{HCO}^{+}\right)$have already been observed, and the other (NO) has been sought but not yet found. Table III presents a comparison between the observed and predicted column densities in a number of molecular clouds. The agreement is quite striking, lending support to the view that gas-phase reactions play a role in making interstellar molecules.

Clouds near $\mathrm{H}_{\mathrm{II}}$ regions are often particularly rich in molecules; the radio object Orion A is an example of such a cloud. Its optical counterpart, the Orion Nebula, appears to be on the near side of a giant dark cloud which is not very apparent in photographs but which is easily detected in the radio region. It is not understood why dark clouds near Hil regions should be so rich in molecules. Perhaps this is an effect of observational selection, because HII regions are caused by the interaction of young stars and interstellar gas, and the formation of young stars presupposes the collapse of interstellar gas to high densities, which favor molecule formation.

\section{Summary}

Our theme is that cores of interstellar dust grains, which are made in and ejected from cool stars, determine the chemical nature of the interstellar medium. Figure 7, our 
last, is a sketch of a hypothetical dust grain, showing a core of silicates and iron, a mantle of ice and other molecules, and a thin layer of adsorbed $\mathrm{H}$ atoms. Hydrogen atoms hitting the grain react with those on the surface to form $\mathrm{H}_{2}$. By the ionmolecule reactions in Figure 6, some of the $\mathrm{H}_{2}$ reacts with $\mathrm{O}$ to form $\mathrm{OH}$ and $\mathrm{H}_{2} \mathrm{O}$.

Oxygen atoms hitting the grain react with $\mathrm{H}$ in the adsorbed layer to become $\mathrm{OH}$. If the $\mathrm{OH}$ is ejected on formation, gaseous $\mathrm{OH}$ is produced. If not, the $\mathrm{OH}$ reacts further with $\mathrm{H}$ on the grain to form $\mathrm{H}_{2} \mathrm{O}$, which becomes part of the mantle. Along with other molecules in the mantle, these are processed by ultraviolet light to become more complex molecules.

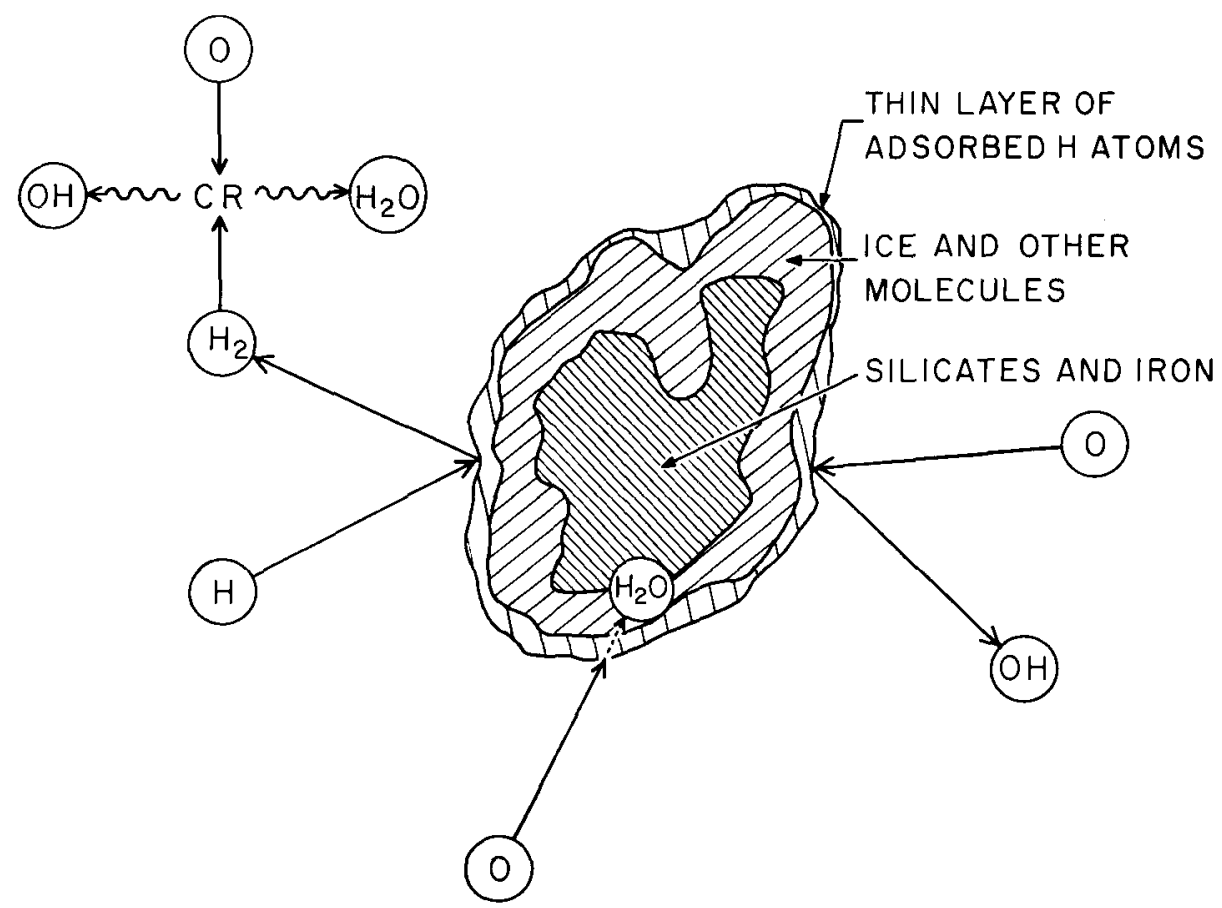

Fig. 7. Sketch of a hypothetical interstellar dust grain, magnified one million times. Surface reactions with $\mathrm{H}$ and $\mathrm{O}$ are shown. Aided by cosmic-ray ionization, the $\mathrm{H}_{2}$ reacts further with $\mathrm{O}$ to form $\mathrm{OH}$ and $\mathrm{H}_{2} \mathrm{O}$. Other elements, such as $\mathrm{C}$ and $\mathrm{N}$, produce other molecules by similar processes.

The net result of these processes is a core-mantle grain which fits the interstellar extinction observations, and gas-phase molecules which fit the radio observations. That atoms are indeed lost to the grains and to molecules is proved by the deficiencies of gas-phase atoms discovered by Copernicus.

In conclusion, I would like to point out an amusing analogy. The dust grain in Figure 7 bears qualitative resemblance to a planet! The interior of the grain, like that of the Earth, is composed of iron and silicates. Its outer envelope, like the oceans of Earth, is water. The whole is immersed in a gaseous atmosphere, and is bathed, 
like the Earth, in ultraviolet light and cosmic radiation. Radio astronomy has shown that the chemical reactions in and around interstellar dust grains produce molecules of surprising complexity. Perhaps there is a lesson here for the chemists who are trying to reconstruct the synthesis of organic molecules which must have preceded life on Earth. While we do not need to postulate life in space to explain interstellar molecules, the processes which are producing them in space may be very similar to those which occurred three billion years ago on primitive Earth.

\section{References}

Bless, R. C. and Savage, B. D.: 1972, Astrophys. J. 171, 293.

Carrasco, L., Strom, S. E., and Strom, K. M.: 1973, Astrophys. J. 182, 95.

Field, G. B.: 1974, Astrophys. J. 187, 453.

Gillett, F. C., Forrest, W. J., and Cohen, M.: 1972, in preparation.

Hackwell, J. A.: 1971, Thesis, University College, London.

Herbst, E. and Klemperer, W.: 1973, Astrophys. J. 185, 505.

Hollenbach, D. J., Werner, M. W., and Salpeter, E. E.: 1971, Astrophys. J. 163, 165.

Lewis, J. S.: 1972, Icarus 16, 241.

Spitzer, L., Drake, J. F., Jenkins, E. B., Morton, D. C., Rogerson, J. B., and York, D. G.: 1973, Astrophys. J. 181, L116. 